\title{
Ganoderic Acid Accumulation and Biosynthetic Gene Expression during Fruiting Body Development in Ganoderma lucidum
}

\author{
Zhao-Rui Feng \\ School of Public Administration \\ Yunnan University \\ Kunming, China \\ e-mail:fzrui86@163.com
}

\author{
Zhao-Rui Feng \\ Quality Development Institute \\ Kunming University of Science and Technology \\ Kunming, China \\ e-mail:fzrui86@163.com
}

\author{
Huan-Jun Li \\ Faculty of Life Science and Technology \\ Kunming University of Science and Technology line 3: \\ Kunming, China \\ e-mail: 562346280@qq.com
}

\author{
Jun-Wei Xu \\ Faculty of Life Science and Technology \\ Kunming University of Science and Technology line 3: \\ Kunming, China \\ e-mail: xjuwei@163.com
}

\begin{abstract}
Objective: This work aimed to investigate the accumulation of total triterpenoids, individual ganoderic acid (GA) content and expression of four important biosynthetic genes during fruiting body development in Ganoderma lucidum. Methods: Individual GA content was determined by high performance liquid chromatography, and the transcription levels of the GA biosynthetic genes were analyzed by real-time quantitative PCR. Results: The maximum content for total triterpenoids, GA-T, GA-S and GA-Me was 1210, 16.5, 14.3, 12.5 g/ $100 \mathrm{mg}$ dry weight, respectively, at the immature stage. The maximum transcript levels for GA biosynthetic genes encoding 3hydroxy-3-methylglutaryl-CoA reductase, farnesyl pyrophosphate synthase, squalene synthase and lanosterol synthase were up-regulated by 1.8-, 8.7-, 30.5- and 19.2-fold at the immature stage. Conclusion: The result showed that the content of total triterpenoids and individual GA at the immature stage was higher than that at the primordium and the mature stages. The higher GA content at the immature stage was partially related to increased transcription of these genes. This work will be help for better utilization of fruiting body of $G$. lucidum and further investigations on the GA biosynthesis regulation.
\end{abstract}

Keywords- Ganoderma lucidum; individual ganoderic acid; total triterpenoids; gene expression; fruiting body development

\section{INTRODUCTION}

Ganoderma lucidum, Ling-zhi in Chinese and Reishi in Japanese, has been used as a folk medicine for the prevention and treatment of various diseases for several thousand years in Asia. Ganoderic acids, as a kind of triterpenoid compounds produced by this medicinal mushroom, have a number of pharmacological activities including cytotoxicity to hepatoma cells, antitumor, antimetastasis, and anti-HIV activities [1]. Recent studies showed that different individual GAs have different bioactivities, for example, GA-S stimulates platelet aggregation [2], GA-T induces apoptosis of lung cancer cells [3], and GA-Me inhibits tumor growth and lung metastasis.

GA is synthesized via the mevalonate/isoprenoid (MVA) pathway, which involves the sequential conversion of farnesyl disphosphate to squalene, 2, 3oxidosqualene, and lanosterol. The steps after lanosterol formation including a series of oxidation, reduction, and acylation reactions to form different individual GA are yet unclear [1]. Several genes involved in early steps of the GA biosynthetic pathway that encode the 3-hydroxy-3methylglutaryl CoA reductase (HMGR), farnesyldiphosphate synthase (FPS), squalene synthase (SQS), and lanosterol synthase (LS) have already been isolated and characterized [5].

The fruiting body of G. lucidum has a significant use history as traditional medicines and health food in China. Many pharmacologically active triterpenoids including GA have been isolated from the fruiting body of $G$. lucidum [6]. Previously, it was reported that the triterpenoid components of extracts isolated from fruiting bodies of various growth stages (small, medium and large) were different in G. lucidum [7]. However, to the best of our knowledge, information regarding the quantitative effect of the developmental stage on total triterpenoid accumulation and individual GA content is not yet available. It was known that the quality of the medicinal mushroom was dependent on the bioactive component content of the fruiting body, and therefore, it is important to determinate bioactive component content at different developmental stages of fruiting body formation either for use on the preparation of pharmalogical or to achieve the ideal stage for consumption concerning pharmaceutical value. 
Zhao et al. cloned HMGR, FPS, SQS and LS gene in G. luciudm, and found that the expression levels of those genes were higher in the primordium than that in the mycelia [8-11]. But, there is yet no information on the expression patterns of GA biosynthetic genes during the fruiting body development of $G$. lucidum in spite of the great need for such knowledge in understanding the regulation of development on secondary metabolism.

In this work, the accumulation of total triterpenoids, individual GA content and expression of GA biosynthetic gene (hmgr, fps, sqs and $l s)$ were analyzed at the different developmental stages of G. lucidum. This work will be help for better utilization of fruiting body of G. lucidum and further investigations on the GA biosynthesis regulation.

\section{MATERIALS AND METHODS}

\section{A. Strain and culture conditions}

The strain of G. lucidum CGMCC 5.616 from China General Microbiological Fermentation Center was maintained on potato dextrose agar slants. The details of preculture conditions were described earlier [12]. For solid-state fermentation, the following substrates were used: $58 \%$ cotton seed hull, $20 \%$ wheat bran, $20 \%$ sawdust, $1 \% \mathrm{CaSO} 4$ and $1 \%$ sucrose. Moisture content was adjusted to $60 \%$ by adding the appropriate quantity of distilled water. Each polypropylene bag was filled with 200 g (dry weight) substrate. All containers were sterilized at $121^{\circ} \mathrm{C}$ for $40 \mathrm{~min}$. After cooling, $10 \%$ inoculum (20 ml inocula for $200 \mathrm{~g}$ solid substrates) was used for each bag. Spawning and fruiting conditions were conducted as described by Hsieh et al. [13].

\section{B. Sampling, extraction and analyses of total triterpenoids and individual GA}

For sampling, three bags were taken each time. Samples were collected at three different developmental stages: the primordium stage (day 9), the immature stage (cap formed, white cap brim not yet fully expanded, day 30) and the mature stage (cap fully developed, yellow growth line absent, day 50). The mushroom was separated into three different parts (ground part, stalk and cap) and dried at $50{ }^{\circ} \mathrm{C}$ to constant weight. Total triterpenoids were extracted from the fruiting body (100 mg DW) by ethanol (3 mL), ultrasonic treatment for $1 \mathrm{~h}$ (three times). Total triterpenoids were measured according to the method described elsewhere [14]. For determining individual GAs, high performance liquid chromatography (HPLC) was performed on an Agilent 1200 series (5 $\mu \mathrm{m}$ Agilent Zorbax SB-C18 column, $250 \times 4.6 \mathrm{~mm}$ ). Details on the HPLC analysis method are described elsewhere [12].

\section{Total RNA extraction and cDNA synthesis}

For sampling, three bags were taken each time. Samples were collected at three different developmental stages: the primordium stage (day 9), the immature stage (cap formed, white cap brim not yet fully expanded, day 30) and the mature stage (cap fully developed, yellow growth line absent, day 50). The mushroom was separated into three different parts (ground part, stalk and cap) and dried at $50{ }^{\circ} \mathrm{C}$ to constant weight. Total triterpenoids were extracted from the fruiting body (100 $\mathrm{mg} \mathrm{DW})$ by ethanol (3 $\mathrm{mL})$, ultrasonic treatment for $1 \mathrm{~h}$ (three times). Total triterpenoids were measured according to the method described elsewhere [14]. For determining individual GAs, high performance liquid chromatography (HPLC) was performed on an Agilent 1200 series (5 $\mu \mathrm{m}$ Agilent Zorbax SB-C18 column, $250 \times 4.6 \mathrm{~mm}$ ). Details on the HPLC analysis method are described elsewhere [12].

D. Measurement of HMGR, FPS, SQS and LS gene expressions by real time quantitative PCR ( $q R T-P C R)$

The transcription levels of the HMGR, FPS, SQS and $L S$ genes were analyzed by real-time quantitative PCR (qRT-PCR) as described by Zhou et al. [15]. The gene for $18 \mathrm{~S}$ rRNA was used as the reference gene. The expression level of the different genes was normalized with respect to the 18S rRNA expression level. For each gene, the reference sample (the ground part on day 1) was defined as the expression level 1.0, and results of other samples were expressed as the fold of mRNA level over the reference sample.

\section{RESULTS AND DISCUSSION}

A. Total triterpenoid accumulation and individual GA $(G A-T, \quad-S, \quad-M e)$ content during fruiting body development in G. lucidum

G. lucidum primordium initialized, along with the mycelia mat (ground part on the surface of the substrate) forming on the day 9 after inoculation. Afterwards, primordium elongated and differentiated into stalk and cap. The fruiting bodies of G. luciudm were harvested and dried at different maturity stages. Fig. 1 showed that total triterpenoid content in different parts (ground part, stipe and cap) of fruiting body at different developmental stages. The total triterpenoid content at all maturity stages ranged from 0.7 to $1.12 \mathrm{mg} / 100 \mathrm{mg}$ DW. The content of total triterpenoids at the immature stage was much higher than that at the primordium and mature stages. The maximum content of total triterpenoids was $1.21 \mathrm{mg} / 100$ mg DW at the immature stage (day 30) in the ground part of the fruiting body. The content of the total triterpenoids in the stipe part and the cap part was 1.13 and 1.15 $\mathrm{mg} / 100 \mathrm{mg} \mathrm{DW}$, respectively, at the immature stage. The contents of total triterpenoids in both the ground part $(0.65 \mathrm{mg} / 100 \mathrm{mg} \mathrm{DW})$ and stipe $(0.7 \mathrm{mg} / 100 \mathrm{mg} \mathrm{DW})$ decreased with the increase of the fruiting body maturity. The results indicated that the developmental stages affect total triterpenoid accumulation in G. lucidum. This is in agreement with Boh B et al. who reported that the amount 

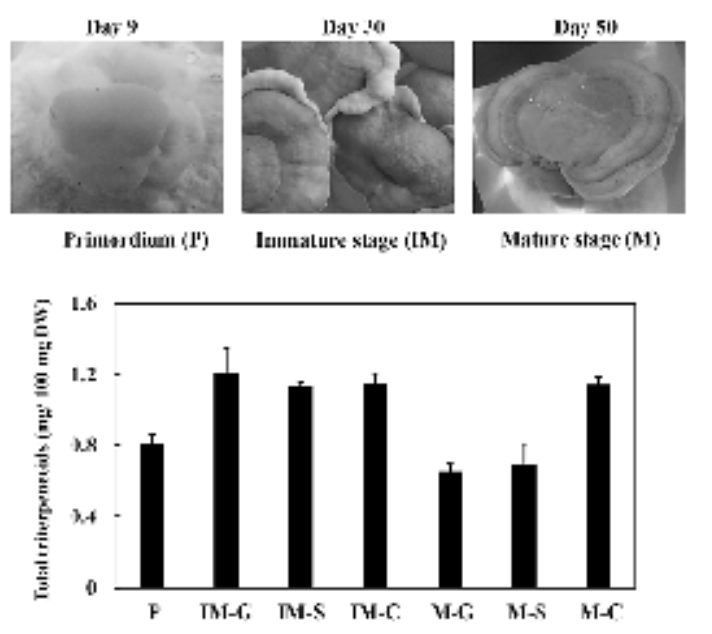

Figure 1: Total triterpenoid accumulation at the different developmental stages of G. lucidum. P, primordium; IM-G, ground part at the immature stage; IM-S, stipe at the immature stage; IM-C, Cap at the immature stage. M-G, ground part at the mature stage; M-S, stipe at the mature stage; M-C, Cap at the mature stage

of triterpenoid acids was higher in younger part of $G$. applanatum fruiting bodies than that in the older part [16]. In Catharanthus pusillus, it also was found that terpenoid level was depended on the development stages of the plants [17].

With regard to the individual GA, the content of three major individual GA (GA-T, -S, -Me) was assayed at the different developmental stages. Individual GA accumulation at the immature stage was significantly higher compared with that at the primordium stage and the mature stage (Fig. 2). This was consistent with total triterpenoid accumulation during fruiting body development in G. lucidum. The maximum contents for GA-T, GA-S and GA-Me were 16.5, 14.3, 12.5 g/ 100 $\mathrm{mg}$ DW in the stipe part at the immature stage. Profile of individual GA content changed during fruiting body development in G. lucidum, which suggested that the biosynthesis of individual GA may correlate with mushroom differentiation. Previously, Nishitoma et al. also reported that the triterpene components of G. lucidum differ in the growing stages accompanied by morphological alternations [7]. As shown in Fig. 2, the distribution of individual GA content was different in different parts of the fruiting bodies of G. lucidum. As a similar case, in Tricholoma masutake, it was found that the content of triterpenes in the stipe part was twofold of those in the cap part [19]. Both total triterpenoid content and individual GA content reached their maximum level at the immature stage. Our results suggested that the fruiting bodies of G. lucidum should be harvested at the immature stage for medicinal purposes in which the content of bioactive component reached their highest level.

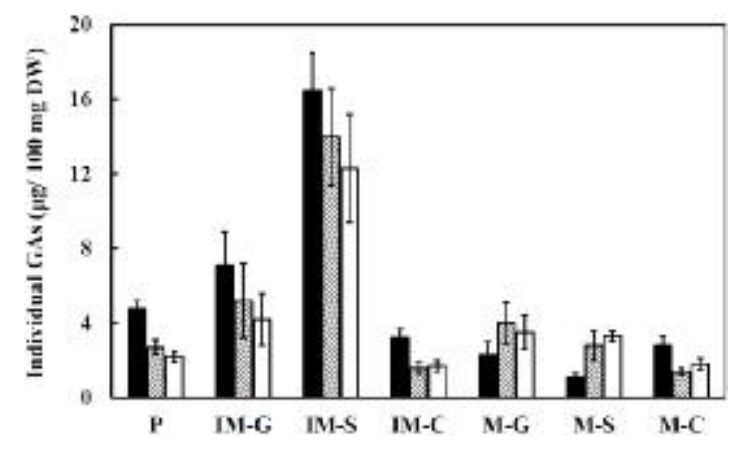

Figure 2: The accumulation of GA-T (dark bar), GA-S (hatched bar) and GA-Me (blank bar) at the different developmental stages of $G$. lucidum

GAs are synthesized through the MVA pathway. To explore the molecular mechanism underlying the changed patterns of total triterpenoids and individual GA observed at different developmental stages, the expression levels of four important genes, i.e., HMGR, SQS, FPS and LS, were examined by qRT-PCR. Gene expression was normalized against the expression level of the 18S rRNA gene. The expression of each gene in the ground part (day 1) was arbitrarily assigned the value of 1.0. The four GA biosynthetic genes showed different transcription accumulation patterns or levels at different developmental stages of G. lucidum (Fig. 3). In previously reports, it was found that GA biosynthetic genes were expressed differentially in mycelia and primordium [8-11]. The difference in the gene expression may be caused by the fruiting body development of $G$. lucidum. Similar observation was also reported for Brassica juncea, in which developmental regulation of HMGR gene occurs in the flower and seed [20]. In grey poplar leaves, it was reported that the transcript level of phytoene synthase is regulated by development of leaf [21].

The maximum transcript levels for hmgr, fps, sqs and $l s$ were 1.8-, 8.7-, 30.5- and 19.2-fold that of the control at the immature stage. Fig. 3 showed that transcription levels of four biosynthetic genes were highest at the immature stage, and declined at the mature stage. The differences in transcription levels of GA biosynthetic genes were approximately agreement with the difference in total triterpenoid accumulation and individual GA content. Our results suggested that the changed triterpenoid accumulation patterns were at least partially due to the different transcription levels of GA biosynthetic genes. In Lavandula angustifolia Mill., it also was found that the changed transcription level of terpene synthase lead to different volatile terpene content during lavender inflorescence development [22].

\section{B. Expression patterns of GA biosynthetic genes at different developmental stages}



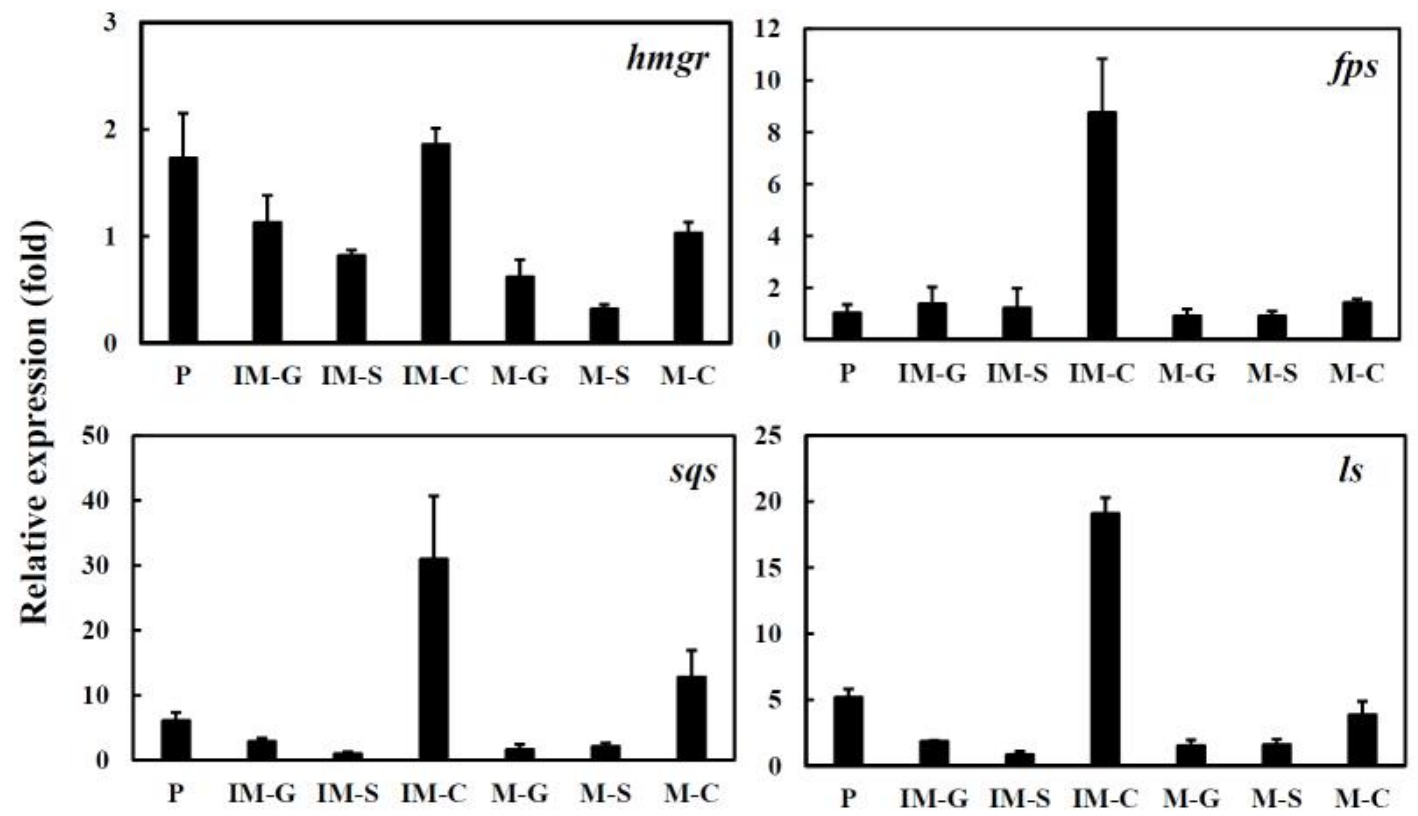

Figure 3: Detection of $h m g r, f p s, s q s$ and $l s$ gene expression at the different developmental stages of G. lucidum

\section{ACKNOWLEDGMENT}

Financial support from the National Natural Science Foundation of China (Project No. 31360495), the start-up grant from Kunming University of Science and Technology (Project No. KKSY201226107), and the Analysis and Measurement Foundation of Kunming University of Science and Technology (Project Nos. 20130468 and 20130433) is gratefully acknowledged.

\section{REFERENCES}

[1] Xu JW, Zhao W, Zhong JJ, Biotechnological production and application of ganoderic acids. Appl Microbiol Biotechnol, 87, 457-466, 2010.

[2] Wang CN, Chen JC, Shiao MS, Wang CT, The aggregation of human platelet induced by ganodermic acid S. Biochim Biophys Acta, 1989; 986,151-160, 1989.

[3] Tang W, Liu JW, Zhao WM, Wei DZ, Zhong JJ, Ganoderic acid T from Ganoderma lucidum mycelia induces mitochondria mediated apoptosis in lung cancer cells. Life Sci, 80,205-211, 2006.

[4] Chen NH, Liu JW, Zhong JJ, Ganoderic acid Me inhibits tumor invasion through down-regulating matrix metalloproteinases 2/9 gene expression. J Pharm Sci, 108, 212-216, 2008.

[5] Shi L, Ren A, Mu DS, Zhao MW, Current progress in the study on biosynthesis and regulation of ganoderic acids. Appl Microbiol Biotechnol, 88,1243-1251, 2010.

[6] Zhou XW, Lin J, Yin YZ, Zhao JY, Sun XF, Tang KX, Ganodermataceae: Natural products and their related pharmacological functions. Am J Chin Med, 35, 559-574, 2007.

[7] Hirotani M, Furuya T, Changes of the triterpenoid patterns during formation of the fruit body in Ganoderma lucidum. Phytochemistry, 29, 3767-3771, 1990

[8] Zhao MW, Liang WQ, Zhang DB, Wang N, Wang CG, Pan YJ, Cloning and characterization of squalene synthase (SQS) gene from Ganoderma lucidum. J Microbiol Biotechnol, 17, 1106-1112, 2007.

[9] Shang CH, Shi L, Ren A, Qin L, Zhao MW, Molecular cloning, characterization, and differential expression of a lanosterol synthase gene from Ganoderma lucidum. Biosci Biotechnol Biochem, 74, 974-978, 2010.

[10] Shang CH, Zhu F, Li N, Ou-Yang X, Shi L, Zhao MW, Li YX, Cloning and characterization of a gene encoding HMG-CoA reductase from Ganoderma lucidum and its functional identification in yeast. Biosci Biotechnol Biochem, 72, 1333-1339, 2008.

[11] Ding YX, Ou-Yang X, Shang CH, Ren A, Shi L, Li YX, Zhao MW, Molecular cloning, characterization, and differential expression of a farnesyl-diphosphate synthase gene from the basidiomycetous fungus Ganoderma lucidum. Biosci Biotechnol Biochem, 72, 1571$1579,2008$.

[12] Xu JW, Xu YN, Zhong JJ, Production of individual ganoderic acids and expression of biosynthetic genes in liquid static and shaking cultures of Ganoderma lucidum. Appl Microbiol Biotechnol, 85, 941-948, 2010.

[13] Hsieh C, Yang FC, Reusing soy residue for the solid-state fermentation of Ganoderma lucidum. Bioresour Technol, 91, 105$109,2004$.

[14] Liu J, Zhang Z, Yang G, Niu H, Content determination of total triterpenes compound and oleanolic acid in Viscum Coloratum and its photosynthetic bacterium transformation products. China Pharm, 22, 1385-1387, 2011.

[15] Zhou JS, Ji SL, Ren MF, He YL, Jing XR, Xu JW, Enhanced accumulation of individual ganoderic acids in a submerged culture of Ganoderma lucidum by the overexpression of squalene synthase gene. Biochem Eng J, 90, 178-183, 2014.

[16] Boh B, Hodzar D, Dolnicar D, Berovic M, Pohleven F, Isolation and quantification of triterpenoid acids from Ganoderma applanatum of Istrian origin. Food Technol Biotechnol, 1, 11-18, 2000.

[17] Zarate R, Dirks C, van der Heijden R, Verpoorte R, Terpenoid indole alkaloid profile changes in Catharanthus pusillus during development. Plant Sci, 160, 971-977, 2001.

[18] Nishitoba T, Sato H, Shirasu S, Sakamura S, Novel triterpenoids from the mycelial mat at the previous stage of fruiting of Ganoderma lucidum. Agric Biol Chem, 51, 619-622, 1987.

[19] Tian GH, Zhou XW, Lin J, Chen WQ, Wang Z, Study on polysaccharide extraction from Tricholoma matsutake fruiting bodies. Amino Acids Biotic Resources, 25, 35-36, 2003.

[20] Alex D, Bach TJ, Chye ML, Expression of Brassica juncea 3hydroxy-3-methylglutaryl CoA synthase is developmentally regulated and stress-responsive. Plant J, 22, 415-426, 2000. 
[21] Mayrhofer S, Teuber M, Zimmer I, Louis S, Fischbach RJ, Schnitzler JP, Diurnal and seasonal variation of isoprene biosynthesis-related genes in grey poplar leaves. Plant Physiol, 139, 474-484, 2005.
[22] Guittona Y, Florence Nicole F, Moja S, Valot N, Legrand S, Jullien F, Legendre L, Differential accumulation of volatile terpene and terpene synthase mRNAs during lavender (Lavandula angustifolia and $L . x$ intermedia) inflorescence development. Physiol Plant, 138, 150-163, 2010. 